\title{
Intussusception of the Appendix and Ileum Endometriosis: A Case Report
}

\author{
Edvinas Dainius $^{a, b} \quad$ Lina Pankratjevaite $^{a}$ Saulius Bradulskis ${ }^{a, b} \quad$ Donatas Venskutonis $^{a, b}$ \\ ${ }^{a}$ Department of Surgery, Medical Academy, Lithuanian University of Health Sciences, Kaunas, Lithuania; \\ ${ }^{b}$ Department of Surgery, Kaunas Clinical Hospital, Kaunas, Lithuania
}

\section{Keywords}

Appendix · Intussusception · Endometriosis

\section{Summary}

Background: Intussusception of the appendix is a very rare condition. It may clinically mimic acute or chronic abdominal diseases or can be asymptomatic. Case Report: This paper describes our experience with intussusception of the appendix. We report a case of a female with appendiceal intussusception and ileum endometriosis. Conclusion: It is important to know about such a rare condition in order to avoid mistaking it with other abdominal diseases. However, diagnosis is rarely made preoperatively.

(C) 2016 S. Karger GmbH, Freiburg

\section{Introduction}

Intussusception is defined as an invagination of one part of the intestine into another section of the bowel. Intussusception of the appendix is a very rare condition. About 200 cases of appendiceal intussusception have been reported in the literature [1]. It was reported first by McKidd [2] in 1858. Intussusception of the appendix can be asymptomatic or may clinically mimic acute or chronic abdominal diseases. Sometimes it can be mistaken with other abdominal diseases. Moreover, diagnosis is rarely made preoperatively. We report a case of a female patient with primary appendiceal intussusception and ileum endometriosis.

\section{Case Report}

A 41-year-old woman presented to the ambulatory with pain in the lower right side of the abdomen and nausea lasting for 3 days. Her past medical history was significant in terms of a right ovariectomy because of a cyst and a hys- terectomy, which was not performed for a cancer. Physical examination revealed an abdominal tenderness in the lower right side of the abdomen. Blood laboratory findings were within normal limits (white blood cells $8.6 \times 10^{9} / 1$, red blood cells $4.38 \times 10^{12} / 1$, hemoglobin $137 \mathrm{~g} / \mathrm{l}$, platelets $\left.348 \times 10^{9} / \mathrm{l}\right)$. An abdominal ultrasound revealed a suspected small bowel intussusception into the cecum of about $2 \mathrm{~cm}$ in length. Radiographic bowel examination with a barium meal test was done; contrast material filled all large bowels after $20 \mathrm{~h}$. Colonoscopy (fig. 1) has shown an edematous Bauhin's valve with small bruising; a biopsy was taken. Histopathological examination of the biopsy material has shown an active ulcerative colitis without tumorous changes. The patient was hospitalized to the Surgery Department 1 week after the illness has started. A computed tomography (CT) scan was performed (fig. 2). It demonstrated small bowel intussusception into the cecum and a contrast filling defect $1.4 \times 1.5 \mathrm{~cm}$ in the lumen of a terminal ileum. After the appropriate counseling, the patient was transferred to the operating room. Lower laparotomy was carried out under combined anesthesia (general and epidural). During the exploration there was no visible appendix in the right iliac fossa, but it was palpated in the cecum (fig. 3). In the terminal ileum, a soft subserosal tumor of about $2 \mathrm{~cm}$ in size was seen approximately $10 \mathrm{~cm}$ from the ileocecal junction. Ileocecal segment resection was performed. The drain was left in the peritoneal cavity. The postoperative period was without any complications. The patient received antibiotic therapy (cefazolin, metronidazole), analgesics, $\mathrm{H}_{2}$ blockers, and low-molecular-weight heparin at prophylactic doses. The wound healed by primary intention.

Histopathological postoperative examination has shown an intussusception of the appendix (fig. 4) with chronic active ulcerative inflammation, hyperplastic/regenerative mucous membrane lesions, and low-grade intraepithelial neoplasia. In addition, small bowel endometriosis was found.

\section{Discussion}

Intussusception of the appendix is a very rare condition with an incidence rate of $0.01 \%$ in a large surgical and autopsy material study [3]. It may occur at any age. Appendiceal intussusception was first described by McKidd [2] in 1858, and up until now only about 200 cases have been reported [1]. Intussusception of the appendix can present itself in different clinical ways, i.e., it may mimic acute or chronic abdominal diseases, or it can be asymptomatic. The symptoms can be similar to acute appendicitis, can be consistent with intestinal intussusception (abdominal pain, vomiting), and sometimes a prolonged history of repeated intermittent

\section{KARGER}

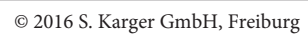




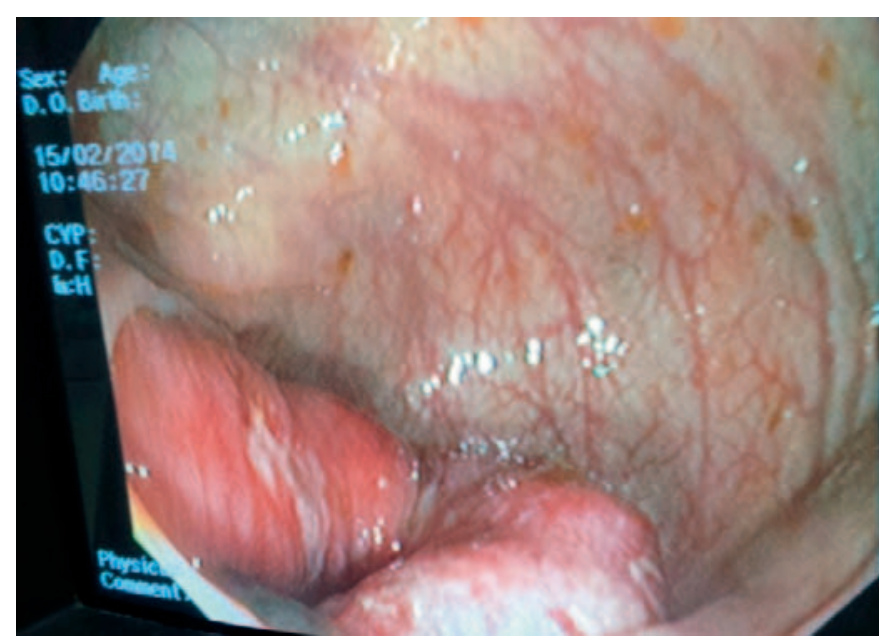

Fig. 1. Colonoscopy showing an edematous Bauhin's valve with small bruising.

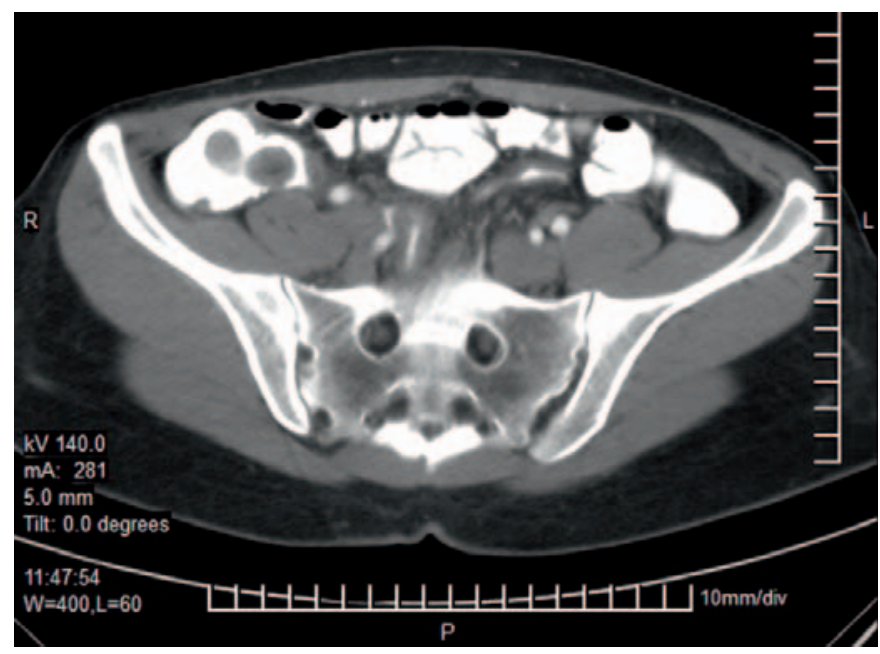

Fig. 2. CT scan demonstrating small bowel intussusception into the cecum and contrast filling defect in the lumen of a terminal ileum.

severe attacks of lower right quadrant abdominal pain can be found. Vomiting and melena may be present, too [4]. In our case, intussusception of the appendix was symptomatic: The patient had a history of pain in the right side of the abdomen and nausea lasting for 1 week. It is interesting that the patient was ill for about 1 week but that the laboratory findings were within normal limits.

Intussusception of the appendix is classified into five anatomic types [5]: type I - invagination of the appendiceal tip; type II - the appendiceal tip is more invaginated to the proximal part of the appendix; type III - intussusception begins at the appendiceal base; type IV - retrograde intussusception; type V - complete appendiceal invagination into the cecum. In our case it was the type last mentioned, i.e. complete appendiceal invagination into the cecum.

The etiology of intussusception of the appendix is mostly unknown $[4,6]$. However, pathophysiological features that determine appendiceal intussusception can be divided into two groups: anatomic and pathologic [4]. Anatomical conditions associated with appendiceal intussusception can be a wide proximal appendiceal lumen, a mobile mesoappendix, and a fetal type of cecum, with the

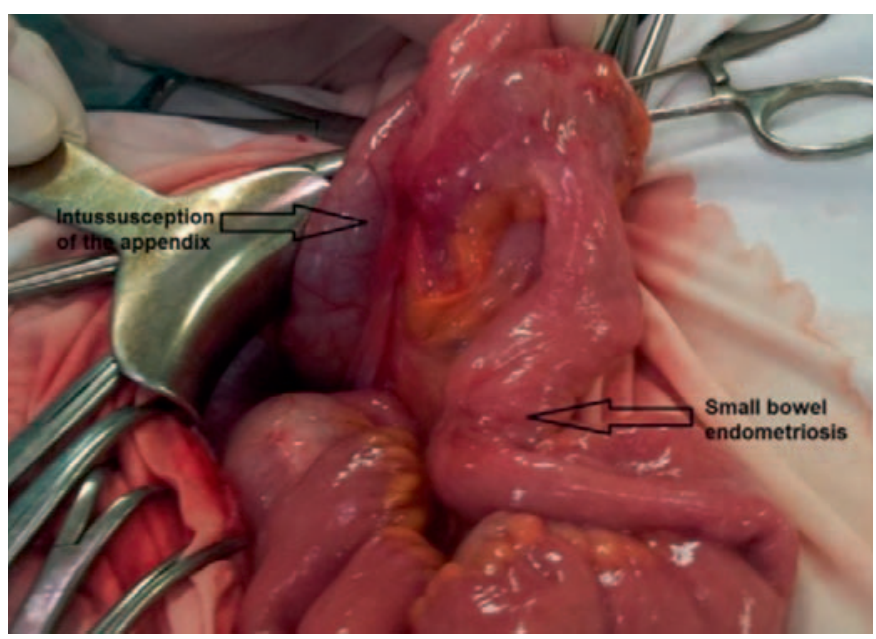

Fig. 3. Operative findings.

appendix originating from its tip [4]. Pathological conditions leading to active peristalsis and appendiceal intussusceptions are various as they can include foreign bodies, fecaliths, parasites, polyps, adenocarcinomas, mucoceles, lymphoid hyperplasia, endometriosis, and so forth [4, 6-8]. The most frequent histopathological finding which comes along with appendiceal intussusception is an inflammation, while the second most common diagnosis is endometrial implants in the intussuscepted appendix [4]. It is important to mention that intussusception of the appendix may occur without any underlying abnormality $[9,10]$. In our case, histopathological examination of surgical material has shown chronic active ulcerative inflammation, hyperplastic/regenerative mucous membrane lesions, and low-grade intraepithelial neoplasia. Low-grade intraepithelial neoplasia (dysplasia) means that neoplasia is present but the risk of developing invasive carcinoma is low [11]. Endometriosis was also found; however, it was in the small bowel and not in the appendix. Endometriosis classically involves the pelvic organs and the pelvic peritoneum. Intestinal involvement, as it was in our case, ranges between 3 and 34\% among women with endometriosis [12].

It is difficult to diagnose appendiceal intussusception prior to the operation. Diagnosis is rarely made preoperatively, and there are just a few cases to be found in the literature in which a preoperative diagnosis of appendiceal intussusception was made $[1,6]$. Appendiceal intussusception can be observed during colonoscopy $[1,6,9,13]$, where it may appear as a sessile or elongated polypoid mass [14]. It is important not to mistake it with a cecal polyp, which can lead to iatrogenic complications after endoscopic removal of such a 'polyp' $[9,10,15]$. Even a simple biopsy of it can provoke bacterial infection [10]. In our case, no intussusception of the appendix was diagnosed during colonoscopy, but an edematous Bauhin's valve with small bruising was seen. There were no complications after biopsy.

Appendiceal intussusception may lessen spontaneously $[7,16]$. The intussusception of the appendix may lessen during colonoscopy, when air is insufflated [17], or there may even be a total reduction of it [4]. If the intussusception of the appendix has been reduced during the colonoscopy, a central depression at the base of 
Fig. 4. Intussusception of the appendix.

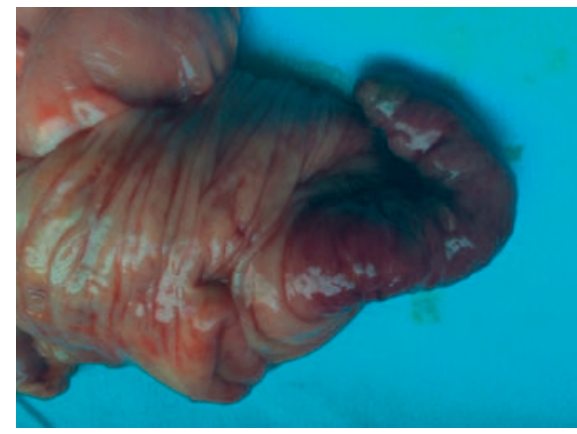

the cecum corresponding to the appendiceal lumen surrounded by an area with an erythematous halo can be seen [1]

Preoperatively, appendiceal intussusception may be diagnosed i) radiographically by means of a barium enema [18], which may show an absence of appendiceal filling and a cecal filling defect [19], ii) by sonography $[20,21]$, where intussusception of the appendix may look like a target or concentric ring [21], or iii) by CT. In our case, an abdominal ultrasound revealed a suspected intussusception. However, it looked like a small bowel intussusception into the cecum and not like an appendiceal intussusception. CT scan demonstrated small bowel intussusception into the cecum, too. No filling defect was seen during the radiographic examination with a barium meal.

The treatment of appendiceal intussusception can be conservative, minimally invasive, and surgical [4]. The intussuscepted appendix may be reduced with a barium enema or air enema. Appendectomy is the treatment of choice in both children and adults [16, 22]. It can be carried out by means of a laparotomy or laparoscopically [4]. Some authors suggest performing an exploratory laparotomy if there is any suspicion of appendiceal mass, cecal neoplasm, or compound ileocecal-appendiceal intussusception [4]. In some situations, ileocecal resection and partial cecectomy could be performed; e.g., if there is concern for a neoplasm, then an ileocecectomy or right hemicolectomy may be necessary [16]. Right hemicolectomy with lymph node resection should be done when the diameter of the tumor is larger than $2 \mathrm{~cm}$ or when malignancy is suspected [4]. In our case, laparotomy and ileocecal segment resection was performed in order to remove the appendix and the tumor (which was an endometrial implant) in the terminal ileum. Naturally, in the case of patients with long-standing asymptomatic appendiceal intussusceptions, some authors suggest to avoid a surgical intervention [17]. However, this pathology is too rare, and there are no reliable data for evaluating the risk of complications in the natural course of an appendiceal intussusception.

\section{Conclusions}

Intussusception of the appendix is a very rare condition. Its clinical presentation varies greatly. There are no specific symptoms which can help to distinguish appendiceal intussusception from other diseases. Moreover, radiological or instrumental examination does not always result in the diagnosis being intussuscepted appendix. It is very important to recognize this condition and to regard appendiceal intussusception as a malignancy in order to avoid inappropriate treatment such as colonoscopic 'polypectomy' or unnecessary hemicolectomy. Furthermore, doctors must know that appendiceal intussusception can be treated conservatively or minimally invasively; however, it is mainly treated surgically.

\section{Disclosure Statement}

The authors declare that they have no conflict of interest.

\section{References}

1 Ozuner G, Davidson P, Church J: Intussusception of the vermiform appendix: preoperative colonoscopic diagnosis of two cases and review of the literature. Int J Colorectal Dis 2000;15;3:185-187.

2 McKidd J: Case of invagination of the caecum and appendix. Edinburgh Med J 1858;4:793-796.

3 Collins DC: Seventy-one thousand human appendix specimens: a final report, summarizing forty years of study. Am J Proctol 1963;14:365-381.

4 Varsamis N, Pouggouras K, Salveridis N, Theodosiou A, Lostoridis E, Karageorgiou G: Appendiceal intussusception; in Lule G (ed): Current Concepts in Colonic Disorders. Rijeka, InTech, 2012, pp 47-64.

5 McSwain B: Intussusception of the appendix: review of the literature and report of a case. South Med J 1941; 34:263-271.

6 Duncan JE, DeNobile JW, Sweeney WB: Colonoscopic diagnosis of appendiceal intussusception: case report and review of the literature. JSLS 2005;9:488-490.

7 Komine N, Yasunaga C, Nakamoto M, Shima I, Iso Y, Takeda Y, Nakamata T: Intussusception of the appendix that reduced spontaneously during follow-up in a patient on hemodialysis therapy. Internal Med 2004; 43;6:479-483.
$>_{8}$ Lee DJ, Kim HC, Yang DM, Kim SW, Ryu JK, Won KY, Lee SH: A case of intussusception of the appendix secondary to endometriosis: US and CT findings. J Clin Ultrasound 2015;43:443-446.

9 Pohl J: Intussusception of the appendix. Video J Encyclopedia GI Endosc 2013;1:377.

10 Seddik H, Rabhi M: Two cases of appendiceal intussusception: a rare diagnostic pitfall in colonoscopy. Diagn Ther Endosc 2011;2011:198984.

11 Schlemper RJ, Riddell RH, Kato Y, et al: The Vienna classification of gastrointestinal epithelial neoplasia. Gut 2000;47:251-255.

12 Moradi P, Barakate M, Gill A, Farrow G: Intussusception of the vermiform appendix due to endometriosis presenting as acute appendicitis. ANZ J Surg 2007;77: $758-760$.

13 Tavakkoli H, Sadrkabir SM, Mahzouni P: Colonoscopic diagnosis of appendiceal intussusception in a patient with intermittent abdominal pain: a case report. World J Gastroenterol 2007;13:31:4274-4277.

14 Khawaja FI: Diseases of the appendix recognized during colonoscopy. Saudi J Gastroenterol 2002;8:43-52.

15 Ryu BY, Kim TH, Jeon JY, Kim HK, Choi YH, Baik GH: Colonoscopic diagnosis of appendiceal intussusception: a case report. J Korean Med Sci 2005;20:680-682.
6 Iqbal CW, Kamath AS, Zietlow SP: Appendiceal intussusception masquerading as an ileocolic intussusception. J Gastrointest Surg 2012;16:1076-1077.

17 Salehzadeh A, Scala A, Simson JNL: Appendiceal intussusception mistaken for a polyp at colonoscopy: case report and review of literature. Ann R Coll Surg Engl 2010;92:6:46-48.

18 DeGerome JH, Rodriguez HP: Primary appendiceal intussusception. Am J Dig Dis 1973;18:704-708.

19 Casey RG, Ofori-Kuma F, Carroll K, Tait R, Farrell P: Intussusception of the appendix by a villous adenoma. Eur J Surg 2001;167:789-791.

20 Maglinte DDT, Fleischer AC, Chua GT, Kelvin FM: Sonography of appendiceal intussusception. Gastrointest Radiol 1987;12:163-165.

-21 Pumberger W, Hormann M, Pomberger G, Hallwirth $\mathrm{U}$ : Sonographic diagnosis of intussusception of the appendix vermiformis. J Clin Ultrasound 2000;28:492496.

22 Alaee A: Reducible appendiceal intussusception: a case report and review the literatures. J Pediatr Rev 2013;1: 70-76. 